\title{
CORRESPONDENCE THEOREMS \\ FOR NONDEGENERATE MODULES AND THEIR ENDOMORPHISM RINGS
}

\author{
ZHOU ZHENGPING
}

(Communicated by Lance W. Small)

\begin{abstract}
Let ${ }_{R} U$ be a left $R$-module whose Morita context is nondegenerate and $S=\operatorname{End}(U)$. We show the following:

(1) There is a projectivity (that is, an order-preserving bijection) between the complement submodules of ${ }_{R} U$ and the complement left ideals of $S$;

(2) $S$ is a left CS ring if and only if ${ }_{R} U$ is a CS module;

(3) $S$ is a Baer and left CS ring if and only if ${ }_{R} U$ is a nonsingular and CS module.

Special cases include some earlier works.
\end{abstract}

\section{INTRODUCTION}

Throughout this paper $R$ and $S$ are associative rings with identity. Modules, unless otherwise specified, are consistently left modules.

Recall that a Morita context $(R, U, V, S)$ consists of two rings $R$ and $S$, two bimodules ${ }_{R} U_{S}$ and ${ }_{S} V_{R}$, and two bimodule homomorphisms (-,-): $U \otimes_{S} V \rightarrow R$ and [-,-]: $V \otimes_{R} U \rightarrow S$ satisfying $u\left[v, u^{\prime}\right]=(u, v) u^{\prime}$ and $v\left(u, v^{\prime}\right)=[v, u] v^{\prime}$ for all $u, u^{\prime} \in U$ and $v, v^{\prime} \in V$ with the images being $I$ and $J$, respectively. $I$ and $J$ are both ideals and are called the trace ideals of the context.

A Morita context $(R, U, V, S)$ is called nondegenerate if $U_{S}$ is faithful and $[V, u] \neq 0$ whenever $0 \neq u \in U$. A module $U$ is called nondegenerate if the derived context $(R, U, V, S)$ is nondegenerate, where $S=\operatorname{End}(U)$ and $V=\operatorname{Hom}(U, R)$. A submodule $X$ of $U$ is said to be a complement in $U$ if $X$ has no proper essential extension in $U$. A module $U$ is called a self-generator if, for any submodule $X$ of $U, X=U F(X)$, where $F(X)=\{s \in \operatorname{End}(U)$, $U s \subseteq X\}$ (cf. $[9,14]$ ); a module $U$ is called a CS module if every complement submodule is a direct summand in $U$; and a ring $S$ is said to be a left (right) $\mathrm{CS}$ ring if ${ }_{S} S\left(S_{S}\right)$ is a CS module. Injective, quasi-injective, semisimple, and uniform modules are all CS modules (cf. [9]); a module $U$ is called nonsingular if, for any $u \in U$ such that $\operatorname{Ann}_{R}(u)$ is essential in $R, u=0$.

In 1989, among main results in [9], Khuri showed that if $U$ is nondegenerate and, in addition, nonsingular (or a self-generator, or a CS module), then there is

Received by the editors August 11, 1992.

1991 Mathematics Subject Classification. Primary 16D80, 16S50.

(C) 1994 American Mathematical Society $0002-9939 / 94 \$ 1.00+\$ .25$ per page 
a projectivity (that is, an order-preserving bijection) between $C(U)$ and $C(S)$, where $C(U)$ and $C(S)$ denote the set of complement submodule of $U$ and $S$, respectively $[9$, Theorems $3.7,3.10]$. Consequently, the author showed that if $U$ is a nondegenerate self-generator or is nondegenerate and nonsingular, then $S$ is a left CS ring if and only if ${ }_{R} U$ is a CS module [9, Corollaries 3.8, 3.11]. The author also showed that if $U$ is a nondegenerate and CS module, then $S$ is a left CS ring [9, Corollary 3.9]. The author obtained her Theorem 3.7 with the help of some kind of Galois connection G2 (cf. [9]) and Theorem 3.10 with different maps from $\mathrm{G} 2$.

Therefore, it is quite natural to ask if we could also get a projectivity between $C(U)$ and $C(S)$ under some weak assumptions, or, even if we fail to establish any projectivity under some weak assumptions, can we still show that $S$ is a left CS ring if and only if ${ }_{R} U$ is a CS module?

In this paper we make use of another kind of correspondence theorem from [12], which is shown more powerful in the situation under consideration, and show that for any nondegenerate context $(R, U, V, S)$ without any additional conditions there is a projectivity between $C(U)$ and $C(S)$ (Theorem 3.4), which contains Theorems 3.7 and 3.10 in [9] as special forms under those stronger conditions on $U$ (Corollaries 3.6, 3.7). A sufficient and necessary condition on $U$ is given so that $S$ is a left CS ring; in particular, if the context is derived from a nondegenerate module $U$, we have that $S$ is a left CS ring if and only if $U$ is a CS module (Theorem 3.8). Some other projectivities and applications are also given. As special cases we get most of Khuri's results on complements in [9]. We also get that $C(U)$ satisfies the a.c.c. (ascending chain condition) (d.c.c (descending chain condition)) if and only if $C(S)$ does, $d(U)<\infty$ if and only if $d(S)<\infty$, and in this case $d(U)=d(S)$, where $d()$ denotes the Goldie dimension (Corollary 3.5).

\section{Preliminaries}

Recall for a Gabriel topology $\tau$ a module $M$ is called $\tau$-torsion if $\operatorname{Ann}_{R}(m)$ $\in \tau$ for every $m \in M$; a module $M$ is called $\tau$-free if $\operatorname{Ann}_{M}(\mathscr{A})=0$ for every $\mathscr{A} \in \tau$; and a submodule $K$ of $M$ is called $\tau$-saturated if $M / K$ is $\tau$-free. For any submodule $K$ of $M, \bar{K}=\{m \in M, \mathscr{A} m \subseteq K$ for some $\mathscr{A} \in \tau\}$ is the smallest $\tau$-saturated submodule of $M$ containing $K$ and $K=\bar{K}$ if and only if $K$ is $\tau$-saturated. If $M$ is $\tau$-torsion and $N$ is $\tau$-free. Then $\operatorname{Hom}(M, N)=0$.

For any two sided ideal $I$ there is a Gabriel topology $\tau$ which is determined by $I$ and $I \in \tau$. From now on, $\tau$ and $\tau^{\prime}$ will denote consistently, unless otherwise specified, the Gabriel topologies determined by the trace ideals $I$ and $J$, respectively. For more details about Gabriel topology or torsion theory, the reader is referred to $[13,5]$.

Also, $K \subseteq_{e} U$ denotes that $K$ is an essential submodule of $U$; i.e., $K \cap K^{\prime} \neq$ 0 if $0 \neq K^{\prime}$ is a submodule of $U$.

First we need the following correspondence theorem [12, Proposition 4].

Proposition 2.1. Let $(R, U, V, S)$ be a Morita context. Then the maps

$$
\begin{gathered}
F: X \mapsto F(X)=\{s \in S, U s \subseteq X\}, \\
F^{-1}: Y \mapsto F^{-1}(Y)=\{u \in U,[V, u] \subseteq Y\}
\end{gathered}
$$

induce a lattice isomorphism between $\operatorname{Sat}_{\tau}(U)$ and $\operatorname{Sat}_{\tau^{\prime}}(S)$, where $\operatorname{Sat}_{\tau}(U)$ 
and $\operatorname{Sat}_{\tau^{\prime}}(S)$ denote the lattices of all $\tau$-saturated submodules of $U$ resp. of all $\tau^{\prime}$-saturated submodules of $S$.

Note that the map $F$ is just the first one used in Galois connection G2 in [9], but $F^{-1}$ is different from the second one $Y \mapsto U Y$ denoted by $S_{U}$ in [9]. However, we can show the following significant relationship between them.

Lemma 2.2. For any left ideal $Y$ of $S$

$$
U Y \subseteq F^{-1}(Y) \subseteq \overline{U Y} \subseteq \overline{F^{-1}(Y)} .
$$

In particular, if $Y \in \operatorname{Sat}_{\tau^{\prime}}(S)$, then $F^{-1}(Y)=\overline{U Y}$.

Proof. $[V, U Y]=[V, U] Y \subseteq Y \Rightarrow U Y \subseteq F^{-1}(Y)$ by the definition. On the other hand, if $u \in F^{-1}(Y)$, then $[V, u] \subseteq Y \Rightarrow U[V, u] \subseteq U Y \Rightarrow(U, V) u=$ $I u \subseteq U Y$ but $I \in \tau$; therefore, $u \in \overline{U Y}$. Finally $U Y \subseteq F^{-1}(Y) \Rightarrow \overline{U Y} \subseteq$ $\overline{F^{-1}(Y)}$.

In particular, if $Y \in \operatorname{Sat}_{\tau^{\prime}}(S)$, then $F^{-1}(Y) \in \operatorname{Sat}_{\tau}(U)$ and $\overline{F^{-1}(Y)}=$ $F^{-1}(Y)$; therefore, $F^{-1}(Y)=\overline{U Y}$.

The following known lemma is also useful in the sequel.

Lemma 2.3. For any Gabriel topology $\tau$ if $M$ is $\tau$-free and $K$ is a submodule of $M$ such that $M / K$ is $\tau$-torsion, then $K \subseteq_{e} M$.

Proof. Let $0 \neq m \in M$. Then there is $\mathscr{A} \in \tau$ such that $\mathscr{A} m \subseteq K$. Since $M$ is $\tau$-free, $\mathscr{A} m \neq 0$. This means $K \subseteq_{e} M$.

\section{CORRESPONDENCE THEOREMS AND APPLICATIONS}

Lemma 3.1. Let $(R, U, V, S)$ be a Morita context. Then

(1) if $U$ is $\tau$-free, then $C(U) \subseteq \operatorname{Sat}_{\tau}(U)$; and

(2) if $S$ is $\tau^{\prime}$-free, then $C(S) \subseteq \operatorname{Sat}_{\tau^{\prime}}(S)$.

Proof. (1) Let $X \in C(U)$. Then $\bar{X} / X$ is $\tau$-torsion. Then $X \subseteq_{e} \bar{X}$ by Lemma 2.3. But $X$ is a complement; therefore, $X=\bar{X}$, i.e., $X \in \operatorname{Sat}_{\tau}(U)$.

(2) By symmetry.

Generally speaking, it is not likely that a complement submodule $X$ of $U$ is $\tau$-saturated for an arbitrary Gabriel topology $\tau$.

Note that $(R, U, V, S)$ is nondegenerate if and only if $U$ is $\tau$-free and $S$ is $\tau^{\prime}$-free [1, Theorem 2]. If the context is derived from a module ${ }_{R} U$, then it is easy to show that the context is nondegenerate if and only if $U$ is $\tau$-free. So we have

Corollary 3.2. Let $(R, U, V, S)$ be nondegenerate. Then $C(U) \subseteq \operatorname{Sat}_{\tau}(U)$, and $C(S) \subseteq \operatorname{Sat}_{\tau^{\prime}}(S)$.

We also need the following lemma.

Lemma 3.3. Let $(R, U, V, S)$ be nondegenerate. Then

(1) if $Y \subseteq Y^{\prime}$ are left ideals of $S$, then $Y \subseteq_{e} Y^{\prime} \Leftrightarrow F^{-1}(Y) \subseteq_{e} F^{-1}\left(Y^{\prime}\right)$; and

(2) if $X \subseteq X^{\prime}$ are submodules of $U$, then $X \subseteq_{e} X^{\prime} \Leftrightarrow F(X) \subseteq_{e} F\left(X^{\prime}\right)$. 
Proof. (1) If $Y \subseteq_{e} Y^{\prime}$, then $U Y \subseteq_{e} U Y^{\prime}$ by the same proof of Proposition 3.2(2) in [9]. Now $U Y \subseteq_{e} F^{-1}(Y) \subseteq_{e} \overline{U Y}$ and $U Y^{\prime} \subseteq_{e} F^{-1}\left(Y^{\prime}\right) \subseteq_{e} \overline{U Y^{\prime}}$ by Lemmas 2.2 and 2.3. Also, $F^{-1}(Y) \subseteq F^{-1}\left(Y^{\prime}\right)$. Therefore, $F^{-1}(Y) \subseteq e$ $F^{-1}\left(Y^{\prime}\right)$. Conversely if $F^{-1}(Y) \subseteq_{e} F^{-1}\left(Y^{\prime}\right)$, then, by Lemmas 2.3 and 2.2, $U Y \subseteq_{e} F^{-1}(Y)$ and $U Y^{\prime} \subseteq_{e} F^{-1}\left(Y\right.$ "). So $U Y \subseteq_{e} U Y^{\prime}$, and hence $Y \subseteq_{e} Y^{\prime}$ by the same proof of Proposition 3.2(2) in [9].

(2) The proof of (2) comes from the same argument as that in Proposition $3.2(4)$ in [9].

Now we are in position to establish our main correspondence theorem.

Theorem 3.4. Let $(R, U, V, S)$ be nondegenerate. Then

$$
F: X \mapsto F(X) \text { and } F^{-1}: Y \mapsto F^{-1}(Y)=\overline{U Y}
$$

form a projectivity between $C(U)$ and $C(S)$.

Proof. It remains to show that $F(X) \in C(S)$ if $X \in C(U)$ and $F^{-1}(Y) \in$ $C(U)$ if $Y \in C(S)$.

First let $F(X) \subseteq_{e} Y$, where $Y$ is a left complement in $S$ (using Zorn's Lemma). Then $X=F^{-1} F(X) \subseteq_{e} F^{-1}(Y)$ by Lemmas 2.1 and 3.3(1). But $X \in C(U)$; hence, $X=F^{-1} F(X)=F^{-1}(Y)$. Also, $Y \in \mathrm{Sat}_{\tau^{\prime}}(S)$ by Lemma 3.2. So $F(X)=Y$ by Lemma 2.1 ; i.e., $F(X) \in C(S)$.

Second let $F^{-1}(Y) \subseteq_{e} X$, where $X$ is a left complement in $U$. Then $Y=F F^{-1}(Y) \subseteq_{e} F(X)$ by Lemmas 2.1 and 3.3(2), but $Y \in C(S)$; hence, $Y=F F^{-1}(Y)=F(X)$. Also, $X \in \operatorname{Sat}_{\tau}(U)$ by Lemma 3.2. So $F^{-1}(Y)=X$ by Lemma 2.1 ; i.e., $F^{-1}(Y) \in C(U)$.

Corollary 3.5. Let $(R, U, V, S)$ be nondegenerate. Then

(1) $C(U)$ satisfies the a.c.c. (d.c.c.) if and only if $C(S)$ does; and

(2) $d(U)<\infty$ if and only if $d(S)<\infty$, and in this case $d(U)=d(S)$.

Proof. (1) is obvious from Theorem 3.4, and (2) follows directly from part (v) of the corollary in [11, p. 52].

Part (2) of the corollary was also shown in [1, Theorem 2].

Corollary 3.6. If $U$ is nondegenerate, then the maps $F$ and $F^{-1}$ form a projectivity between $C(U)$ and $C(S)$, where $S=\operatorname{End}(U)$.

If $U$ is not only nondegenerate but also a self-generator or CS module, then one can show, as in [9], that these additional conditions just make $U X=\overline{U X}=$ $F^{-1}(X)$ for any $X \in C(S)$. Therefore, the above corollary contains Khuri's result as a special case [9, Theorem 3.7].

Recall that when $U$ is nonsingular to any submodule $X$ of $U$, there corresponds a unique complement $X^{e}$ in $U$ such that $X \subseteq_{e} X^{e}$ (cf. [4, p. 61, Proposition 7]). Now we have

Corollary 3.7. Let $(R, U, V, W)$ be nondegenerate and $U$ nonsingular. Then $X \mapsto F(X)$ and $Y \mapsto(U Y)^{e}$ determine a projectivity between $C(U)$ and $C(S)$. Proof. We show that $(U Y)^{e}=\overline{U Y}=F^{-1}(Y)$. By Lemma 2.3, $U Y \subseteq_{e} \overline{U Y}$. Therefore, $\overline{U Y} \subseteq_{e}(U Y)^{e}$, but $\overline{U Y} \in C(U)$ if $Y \in C(S)$; hence, $\overline{U Y}=$ $(U Y)^{e}$.

This corollary contains Theorem 3.10 of [9] as a special case when the context is derived from a nondegenerate and nonsingular module $U$. 
As a main application of Theorem 3.4 we get the following necessary and sufficient condition so that $S$ is a left CS ring.

Theorem 3.8. Let $(R, U, V, S)$ be nondegenerate. Then $S$ is a left $C S$ ring if and only if, for any $X \in C(U), X=U e$ for some $e^{2}=e \in S$.

In particular, if $S=\operatorname{End}(U)$, then $S$ is a left CS ring if and only if $U$ is a CS module.

Proof. $\Rightarrow$ for any $X \in C(U), X=F^{-1} F(X)$ and $F(X) \in C(S)$. If $S$ is a left $\mathrm{CS}$ ring, then $F(X)=S e$ for some $e^{2}=e \in S$. Therefore, $X=F^{-1}(\mathrm{Se})=$ $\overline{U S e}=\overline{U e}$, but it is easy to see that $U e$ is a direct summand of $U$; hence, $U e \in C(U) \subseteq \operatorname{Sat}_{\tau}(U)$. Therefore, $X=\overline{U e}=U e$.

$\Leftarrow$ For any $Y \in C(S), Y=F\left(F^{-1}(Y)\right)$, and $F^{-1}(Y) \in C(U) \Rightarrow Y=$ $F(U e)$ for some $e^{2}=e \in S \Rightarrow Y=\{s \in S, U s \subseteq U e\}=S e$.

When $S=\operatorname{End}(U), X=U e$ for some $e^{2}=e \in S \Leftrightarrow X$ is a direct summand of $U$ [2].

When the context is derived from a nondegenerate module $U$, the theorem contains Corollaries 3.8, 3.9, and 3.11 of [9] as special cases.

In [9, Theorem 3.5] it is shown that if $U$ is nondegenerate, then the maps $X \mapsto F(X)$ and $Y \mapsto U Y$ determine a projectivity between $S_{2}=\{X \subseteq$ $U, X=U F(X), F(X) \in C(S)\}$ and $C(S)$. However, we can show that there is a projectivity between $S_{2}$ and $C(U)$. In fact, we have the following more general result.

Theorem 3.9. Let $(R, U, V, S)$ be a Morita context. Then

$$
F_{1}: X \mapsto \bar{X} \text { and } F_{1}^{-1}: X \mapsto U F(X)
$$

form a projectivity between $S_{1}=\left\{U Y, Y \in \operatorname{Sat}_{\tau^{\prime}}(S)\right\}$ and $\operatorname{Sat}_{\tau}(U)$.

Proof. Let $U Y \in S_{1}$. Then $F_{1}^{-1} F_{1}(U Y)=F_{1}^{-1}(\overline{U Y})=F_{1}^{-1} F^{-1}(Y)=$ $U F F^{-1}(Y)=U Y$. On the other hand, if $X \in \operatorname{Sat}_{\tau}(U)$, then $F_{1} F_{1}^{-1}(X)=$ $F_{1}(U F(X))=\overline{U F(X)}=F^{-1} F(X)=X$.

Theorem 3.10. Let $(R, U, V, S)$ be a Morita context. Then the maps $X \mapsto$ $F(X)$ and $Y \mapsto U Y$ determine a projectivity between $S_{1}$ and $\operatorname{Sat}_{\tau^{\prime}}(S)$.

Proof. By Theorem 3.9 and Proposition 2.1, it remains to show that $F(U Y)=$ $F F_{1}(U Y)=Y$. Let $s \in F(U Y)$. Then $U s \subseteq U Y \Rightarrow[V, U s]=[V, U] s \subseteq$ $[V, U] Y \subseteq Y$. Since $J=[V, U] \in \tau^{\prime}, s \in \bar{Y}=Y$ and $F(U Y) \subseteq Y$. On the other hand, $Y \subseteq F(U Y)$ is clear by the definition of $F$, so $F(U Y)=Y$.

Corollary 3.11. Let $(R, U, V, S)$ be a Morita context with $S$ being $\tau^{\prime}$-free. Then

(1) the above maps determine a projectivity between $S_{2}^{\prime}=\{U Y, Y \in C(S)\}$ and $C(S)$; and

(2) the above maps determine a projectivity between $S_{3}=\{U Y, Y \in L(S)\}$ and $L(S)$, where $L(S)$ denotes the set of left annihilator ideals of $S$.

Proof. (1) follows directly from the fact that $C(S) \subseteq \operatorname{Sat}_{\tau^{\prime}}(S)$.

(2) It suffices to show that $L(S) \subseteq \operatorname{Sat}_{\tau^{\prime}}(S)$. Let $s \in \bar{Y}, Y=\operatorname{Ann}_{s}(Z) \Rightarrow$ $\mathscr{B} s \subseteq Y$ for some $\mathscr{B} \in \tau^{\prime} \Rightarrow(\mathscr{B} s) Z=\mathscr{B}(s Z)=0 \Rightarrow s Z=0$ since $S$ is $\tau^{\prime}$-free $\Rightarrow s \in Y \Rightarrow Y=\bar{Y}$. 
This contains Theorem 3.5 of [9] as a special case, as we will see from the following

Corollary 3.12 [9, Theorem 3.5]. If $U$ is nondegenerate, then the maps $X \mapsto$ $F(X)$ and $Y \mapsto U Y$ determine a projectivity between $S_{2}$ and $C(S)$.

Proof. By the previous corollary it remains to show that $S_{2}=S_{2}^{\prime}$.

If $U Y \in S_{2}^{\prime}$, then $F(U Y)=Y$ from the proof of Theorem 3.10, so $U Y=$ $U F(U Y)$ and $F(U Y)=Y \in C(S)$; i.e., $S_{2}^{\prime} \subseteq S_{2}$, while $S_{2} \subseteq S_{2}^{\prime}$ is obvious from the definition.

Recall that a ring $S$ is said to be left upper (lower) Levitzki if it satisfies the a.c.c. (d.c.c.) on $L(S)$. Right Levitzki rings are defined analogously. Also recall that a ring $S$ is called Baer if, for any $Y \in L(S), Y=S e$ for some $e^{2}=e \in S$. Therefore, from the above correspondence theorems, we have

Theorem 3.13. Let $(R, U, V, S)$ be a Morita context with $S$ being $\tau^{\prime}$-free. Then

(1) $S$ is a CS ring $\Leftrightarrow$ for any $X \in S_{2}, X=U e$ for some $e^{2}=e \in S$; and

(2) $S$ is a Baer ring $\Leftrightarrow$ for any $X \in S_{3}, X=U e$ for some $e^{2}=e \in S$.

In particular, if $S=\operatorname{End}(U)$, these conditions are equivalent to that every element in $S_{2}$, resp. $S_{3}$, is a direct summand of $U$.

(3) $S$ is left upper (lower) Levitzki $\Leftrightarrow S_{3}$ satisfies the a.c.c. (d.c.c.).

(4) $S$ is right upper (lower) Levitzki $\Leftrightarrow S_{3}$ satisfies the d.c.c. (a.c.c.).

The proofs of (1) and (2) are almost the same as that in Theorem 3.8, and the proof of (3) follows from Corollary 3.11(2), while (4) follows from (3) when one notices that $L(S)$ satisfies the a.c.c. (d.c.c.) $\Leftrightarrow R(S)$, the set of right annihilator ideals of $S$, satisfies the d.c.c. (a.c.c.).

Part (1) contains Theorem 3.6(1) of [9] as a special case when the context is derived from nondegenerate $U$.

A ring $S$ is called left Goldie if it satisfies the a.c.c. on $L(S)$ and $C(S)$. Hence we have

Corollary 3.14. Let $(R, U, V, S)$ be a Morita context with $S$ being $\tau^{\prime}$-free. Then if $\operatorname{Sat}_{\tau}(U)$ satisfies the a.c.c., then $S$ is left Goldie.

Proof. Under this assumption, $L(S)$ and $C(S) \subseteq \mathrm{Sat}_{\tau^{\prime}}(S)$ from the proof of Corollary 3.11(2). Then the rest follows from Proposition 2.1 .

In Theorem 3.6(2) in [9], it is shown that if $U$ is nondegenerate and satisfies the a.c.c. on $l(U)=\{U Y, Y \subseteq S\}$, then $S$ is a left Goldie ring. However, this condition implies that $\operatorname{Sat}_{\tau}(U)$ satisfies the a.c.c., as we show.

Proposition 3.15. Let $(R, U, V, S)$ be a Morita context and $l(U)$ satisfy the a.c.c. Then $\operatorname{Sat}_{\tau}(U)$ satisfies the a.c.c.

Proof. Let $X_{1} \subseteq X_{2} \subseteq \cdots$ be an ascending chain of elements in $\operatorname{Sat}_{\tau}(U) \Rightarrow$ $U F\left(X_{1}\right) \subseteq U F\left(X_{2}\right) \subseteq \cdots$ is an ascending chain of elements in $l(U) \Rightarrow U F\left(X_{n}\right)$ $=U F\left(X_{n+i}\right)$ for some $n$ and any $i$ by the a.c.c. on $l(U) \Rightarrow \overline{U F\left(X_{n}\right)}=$ $\overline{U F\left(X_{n+i}\right)}$, but $\overline{U F\left(X_{n}\right)}=X_{n}$ and $\overline{U F\left(X_{n+i}\right)}=X_{n+i}$ by Proposition 2.1 $\Rightarrow X_{n}=X_{n+i}$ for some $n$ and any $i$.

Recall that a ring is called left Utumi if for any left ideal $Y$ the fact that its right annihilator in $S$ is zero implies that $Y \subseteq_{e} S$ [8]. 
Theorem 3.16. If $U$ is nondegenerate, then the following are equivalent.

(1) $U$ is a nonsingular and CS module.

(2) $S$ is a left nonsingular and left CS ring.

(3) $S$ is a Baer and left Utumi ring.

(4) $S$ is a Baer and left CS ring.

(5) $S$ is a left and right nonsingular, left CS ring.

Proof. (1) $\Leftrightarrow$ (2) follows from Theorem 2.1 of [6] and Theorem 3.8.

(2) $\Leftrightarrow$ (3) follows from Theorem 2.1 of [3].

(4) $\Rightarrow(2)$ is clear since Baer rings are both left and right nonsingular.

(2) $\Rightarrow(4)$ It suffices to show $L(S) \subseteq C(S)$. Let $Y=\operatorname{Ann}_{s}(Z) \in L(S)$ and $Y \subseteq_{e} Y^{\prime}$. Then $Y z=0$ for any $z \in Z$. However, $S$ is left nonsingular; we have $Y^{\prime} z=0$, i.e., $Y^{\prime} \subseteq Y=\operatorname{ann}_{s}(Z) \Rightarrow Y^{\prime}=Y \in C(S)$.

(4) $\Rightarrow(5) \Rightarrow(2)$ is obvious.

In [9] Corollary 3.15 showed $(1) \Rightarrow(4)$.

Finally, it is worthwhile to list some classes of nondegenerate modules which have been investigated by some authors:

(1) any generators, which include free modules;

(2) any nonsingular module ${ }_{R} U$ with its trace ideal essential $R$;

(3) torsionless modules over a semiprime ring $R$;

(4) any projective module containing a unimodular element [10] or, more generally, any module ${ }_{R} U$ with $u \in I u$ for all $u \in U$, where $I$ is the trace ideal of $U$ [7].

Therefore, for any class of the above four nondegenerate modules we can apply the related results in this paper, for example,

Corollary 3.17. If $U$ is in any class of the above four and $S=\operatorname{End}(U)$, then

(1) The maps $F$ and $F^{-1}$ form a projectivity between $C(U)$ and $C(S)$.

(2) $S$ is a CS ring if and only if $U$ is a CS module.

Moreover, the following are equivalent.

(3) $U$ is a nonsingular and CS module.

(4) $S$ is a left nonsingular and left CS ring.

(5) $S$ is a Baer and left Utumi ring.

(6) $S$ is a Baer and left CS ring.

(7) $S$ is a left and right nonsingular, left CS ring.

\section{REFERENCES}

1. S. A. Amitsur, Rings of quotients and Morita contexts, J. Algebra 17 (1971), 273-298.

2. F. W. Anderson and K. R. Fuller, Rings and categories of modules, Springer-Verlag, New York, Heidelberg, and Berlin, 1974.

3. A. W. Chatters and S. M. Khuri, Endomorphism rings of modules over nonsingular CS rings, J. London Math. Soc. (2) 21 (1980), 434-444.

4. C. Faith, Lectures on injective modules and quotient rings, Lecture Notes in Math., vol. 246, Springer-Verlag, Heidelberg, 1972.

5. J. S. Golan, Torsion theory, Longman Sci. Tech., Essex, 1986.

6. J. Hutchinson and J. Zelmanowitz, Quotient rings of endomorphism rings of modules with zero singular submodule, Proc. Amer. Math. Soc. 35 (1972), 16-20.

7. J. Hutchinson, Endomorphism rings of torsionless modules, Comm. Algebra 15 (1987), 1921-1927. 
8. S. M. Khuri, Properties of endomorphism rings of modules and their duals, Proc. Amer. Math. Soc. 96 (1986), 553-559.

9. Correspondence theorems for modules and their endomorphism rings, J. Algebra 122 (1989), 380-396.

10. B. R. MacDonald, Endomorphism rings of infinitely generated projective modules, J. Algebra 45 (1977), 69-82.

11. J. C. McConnell and J. C. Robson, Noncommutative noetherian rings, Wiley, New York, 1987.

12. B. J. Müller, The quotient category of a Morita context, J. Algebra 28 (1974), 389-407.

13. B. Stenström, Rings of quotients, Springer-Verlag, Berlin, Heidelberg, and New York, 1975.

14. B. Zimmerman-Huisgen, Endomorphism rings of self-generators, Pacific J. Math. 61 (1975), 587-602.

Department of Mathematics, University of Iowa, Iowa City, Iowa 52242

E-mail address: zhou@math.uiowa.edu 